\title{
Effect of Electric Field on ANTA
}

\section{Lemi Türker}

Department of Chemistry, Middle East Technical University, Üniversiteler, Eskişehir Yolu No: 1, 06800 Çankaya/Ankara, Turkey; e-mail: 1turker@gmail.com; lturker@metu.edu.tr

\begin{abstract}
The present study considers an insensitive explosive, ANTA, (5(3)-amino-3(5)-nitro-1H1,2,4-triazole) which is optimized within the restrictions of DFT (B3LYP/cc-PVTZ and B3LYP/6-311++G(d,p)). The optimized structure is subsequently subjected to singlepoint semi empirical MNDO and/or PM3 level of calculations to visualize the effect of electric field which has been set to magnitudes of 0.001 and 0.01 au. Perturbations on the energy and dipole moment are investigated. Also, the directional effect of the field along the axes of inertia of the molecule has been investigated. Generally, the effect of the field is more pronounced along the principle axis of ANTA.
\end{abstract}

\section{Introduction}

Among various molecular properties, molecular hyperpolarizabilities, namely $\beta$ and $\gamma$ have attracted special attention of scientists for couple of decades. When light is incident on a material, the optical electric field, E, results in a polarization $\mathrm{P}$ of the material. The polarization can be expressed as the sum of the linear polarization component, $\mathrm{P}_{\mathrm{L}}$ and nonlinear polarization $\mathrm{P}_{\mathrm{NL}}[1-5]$.

$$
\begin{aligned}
& \mathrm{P}=\mathrm{P}_{\mathrm{L}}+\mathrm{P}_{\mathrm{NL}} \\
& \mathrm{P}_{\mathrm{L}}=\chi^{(1)} \cdot \mathrm{E} \\
& \mathrm{P}_{\mathrm{NL}}=\chi^{(2)} \cdot \mathrm{EE}+\chi^{(3)} \cdot \mathrm{EEE}+\ldots .
\end{aligned}
$$

The susceptibility tensors $\chi^{(\mathrm{n})}$ give the correct relationship for the macroscopic material. For individual molecules, the polarizability $\alpha$, hyperpolarizability $\beta$, and second

Received: January 20, 2021; Accepted: March 2, 2021

Keywords and phrases: ANTA, 3-amino-5-nitro-1,2,4-triazole, explosive, polarizability, electric field.

Copyright (C) 2021 Lemi Türker. This is an open access article distributed under the Creative Commons Attribution License, which permits unrestricted use, distribution, and reproduction in any medium, provided the original work is properly cited. 
hyperpolarizability $\gamma$ are defined as certain tensor quantities. The susceptibility tensors are weight averages of the molecular values.

Energy of polarizable molecule in a field $\mathrm{E}$ is $[3,4]$;

$$
\varepsilon(E)=\varepsilon^{(0)}-\mu^{(0)} E-\alpha E^{2} / 2 !-\beta E^{3} / 3 !+\ldots
$$

Whereas dipole moment in a field $\mathrm{E}$ is,

$$
\mu=\mu^{(0)}+\alpha \mathrm{E}+\beta \mathrm{E}^{2} / 2 !+\ldots
$$

The polarizabilities can be formulated as derivatives of the dipole moment with respect to incident electric field [1].

$$
\begin{aligned}
\alpha_{i j} & =\left(\delta \mu_{\mathrm{i}} / \delta \mathrm{E}_{\mathrm{j}}\right)_{\mathrm{E}->0} \\
\beta_{\mathrm{ijk}} & =\left(\delta^{2} \mu_{\mathrm{i}} / \delta \mathrm{E}_{\mathrm{j}} \delta \mathrm{E}_{\mathrm{k}}\right)_{\mathrm{E}->0} \\
\gamma_{\mathrm{ijk} \mathrm{l}} & =\left(\delta^{3} \mu_{\mathrm{i}} / \delta \mathrm{E}_{\mathrm{j}} \delta \mathrm{E}_{\mathrm{k}} \delta \mathrm{E}_{\mathrm{l}}\right)_{\mathrm{E}->0}
\end{aligned}
$$

Examining these definitions, one can get various conclusions that molecules with a center of inversion will have no hyperpolarizability $(\beta=0)$ whereas molecules with a large dipole moment and a means for electron density shift will have large hyperpolarizability [1]. In the literature there are various articles and levels of theoretical and experimental research about the polarizabilities and nonlinear optical behavior of certain materials $[2$, 6-12].

5-Amino-3-nitro-1H-1,2,4-triazole (also named as 3-amino-5-nitro-1H-1,2,4-triazole) known as ANTA or ANT is a very insensitive explosive molecule [13]. Its synthesis were described decades ago [14-16]. In addition to its potential use as an insensitive high explosive, ANTA has been shown to be a useful intermediate for the preparation of other explosives. A computational study has been reported on ANTA [17].

In the present study, ANTA molecule has been subjected to electric field of different strengths and the resultant polarization effects have been investigated.

\section{Method of Calculation}

The initial geometry optimizations of all the structures leading to energy minima were achieved by using MM2 method followed by semi-empirical PM3 self-consistent fields molecular orbital (SCF MO) method $[18,19]$ at the restricted level [20]. Then, the structure optimizations have been achieved within the framework of Hartree-Fock (HF) and finally by using density functional theory (DFT) at the levels of B3LYP/CC-PVTZ 
and B3LYP/6-311++G(d,p) (restricted closed-shell) [21, 22]. Note that the exchange term of B3LYP consists of hybrid Hartree-Fock and local spin density (LSD) exchange functions with Becke's gradient correlation to LSD exchange [23]. The correlation term of B3LYP consists of the Vosko, Wilk, Nusair (VWN3) local correlation functional [24] and Lee, Yang, Parr (LYP) correlation correction functional [25]. The normal mode analysis for each structure yielded no imaginary frequencies for the $3 N-6$ vibrational degrees of freedom, where $N$ is the number of atoms in the system. This indicates that the structure of each molecule corresponds to at least a local minimum on the potential energy surface. Furthermore, all the bond lengths were thoroughly searched in order to find out whether any bond cleavage occurred or not during the geometry optimization process. All these computations were performed by using SPARTAN 06 [26].

The calculations related to polarizability have been done by means of single point semi- empirical MNDO and PM3 type calculations based on the optimized structures obtained by density functional approach at the levels of B3LYP/cc-PVTZ and B3LYP/6$311++G(d, p)$. The directional effect of electric field along the axes of inertia of ANTA molecule have been searched by using Hyperchem 06 program [27].

\section{Results and Discussion}

Asymmetric substitution of a conjugated chain with a donor group on one end and an acceptor group on the other end provides the noncenterosymmetry required for a secondorder nonlinearity [28-33]. Since, only the time averaged asymmetrically induced polarization leads to second order nonlinear optical (NLO) effects, only molecules and materials lacking a center of symmetry can exhibit them [28]. The assumption is frequently made that the atoms or molecules are independently polarized by the light with no interatomic or intermolecular coupling [28]. ANTA molecule fulfils that condition. The optimized structure belongs to $\mathrm{C} 1$ point group (at both levels of calculations). Figure 1 shows the optimized structure of ANTA (B3LYP/6-311++G(d,p)). The B3LYP/ccPVTZ level of calculation also leads to very similar optimized structure. Nonlinear polarization becomes more important with increasing field strength. Under normal conditions $\alpha \mathrm{E}>\beta \mathrm{E}>\gamma \mathrm{E}$ where $\alpha, \beta$ and $\gamma$ refer to polarizability, the first and second order polarizabilities, respectively.

When an electric field (EF) is applied, the electron distribution and molecular geometry are disturbed. The atomic polarizability arises due to geometrical disturbance. It is significantly smaller than the electronic polarizability. Note that orientation 
polarizability arises when the molecules have a permanent dipole moment. ANTA has the dipole moment of 8.42 debye (B3LYP/6-311++G(d,p)). Highly polarizable molecules respond strongly to the application of the field [3]. They become highly polarized and the centroid of negative charge is displaced [3].

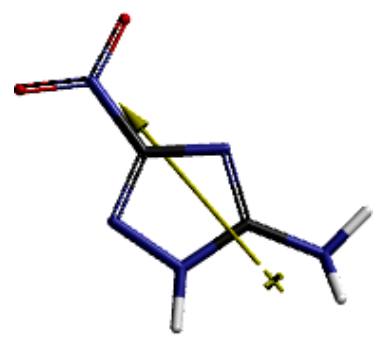

Figure 1. Optimized structure of ANTA (B3LYP/6-311++G(d,p)).

Figure 2 shows the bond lengths of ANTA molecule at two different level of calculations. The lengths differ only very slightly at two different level of calculations. In the present study value of the applied field is kept rather low just to prevent any disturbances of the molecular geometry.
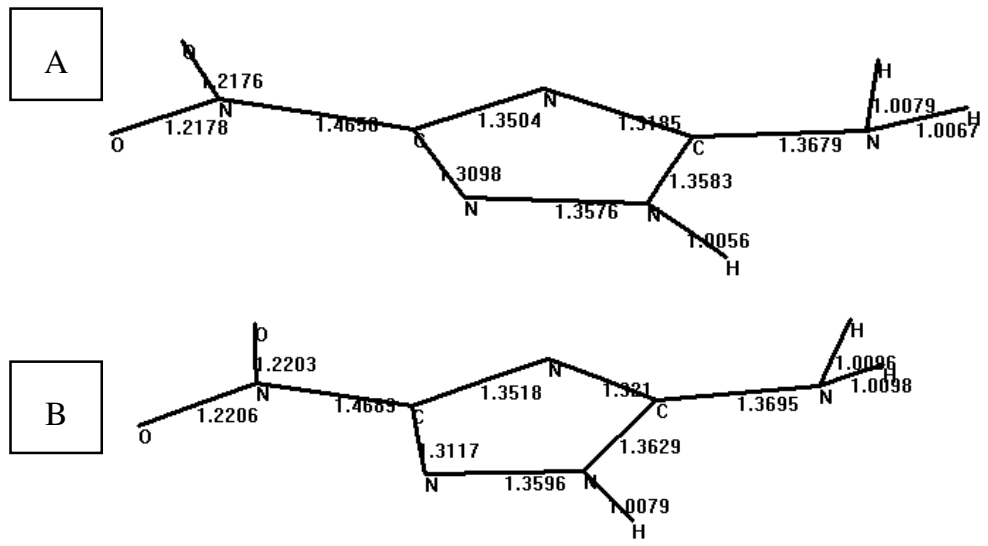

Figure 2. Bond lengths $(\AA)$ in ANTA. A: B3LYP/cc-PVTZ, B: B3LYP/6-311++G(d,p).

Tables $1 \mathrm{~A}$ and $1 \mathrm{~B}$ show the magnitude of dipole moment components as the polarization value increases ten times. The results indicate that the resultant magnitudes decrease as the polarization increases. This behavior holds for every level of calculations presented in the Tables $1 \mathrm{~A}$ and $1 \mathrm{~B}$. Note that in the case of P:0.001 the individual components show parallelism in sign irrespective of the kind of method but when the polarizability value is increased to $\mathrm{P}: 0.01$ that property is lost, especially in the $\mathrm{z}$ component. 
The results of calculations related to polarizability of ANTA molecule are included in Tables 1-5.

Table 1A. Magnitude of dipole moment components (Applied electric field magnitude : $0.001)$.

\begin{tabular}{ccccc}
\hline \multicolumn{5}{c}{ Method } \\
\hline $\begin{array}{c}\text { Field } \\
\text { direction }\end{array}$ & $\begin{array}{c}\text { MNDO// } \\
\text { B3LYP/CC- } \\
\text { PVTZ }\end{array}$ & $\begin{array}{c}\text { PM3// } \\
\text { B3LYP/CC- } \\
\text { PVTZ }\end{array}$ & $\begin{array}{c}\text { MNDO// } \\
\text { B3LYP/6- }\end{array}$ & $\begin{array}{c}\text { PM3// } \\
\text { B3LYP/6- }\end{array}$ \\
\hline $\mathrm{X}$ & -2.857011 & -3.144356 & -2.877493 & $311++\mathrm{G}(\mathrm{d}, \mathrm{p})$ \\
\hline $\mathrm{Y}$ & -0.551770 & -0.672482 & -0.540167 & -3.162892 \\
$\mathrm{Z}$ & 0.456266 & 0.373758 & 0.442735 & -0.662510 \\
\hline \multirow{2}{*}{ Magnitude } & 2.945359 & 3.237113 & 2.961040 & 0.364013 \\
& $(7.485816)$ & $(8.227327)$ & $(7.525671)$ & 3.251970 \\
\hline
\end{tabular}

In au units, Debye units in parenthesis.

Table 1B. Magnitude of dipole moment components (Applied electric field magnitude : $0.01)$.

\begin{tabular}{ccccc}
\hline \multicolumn{5}{c}{ Method } \\
\hline \multirow{2}{*}{ Field } & MNDO// & PM3// & MNDO// & PM3// \\
direction & B3LYP/CC- & B3LYP/CC- & B3LYP/6- & B 3 LP/6- \\
& PVTZ & $311++G(d, p)$ & $311+\mathrm{G}(\mathrm{d}, \mathrm{p})$ \\
\hline $\mathrm{X}$ & 2.855824 & 3.143499 & 2.876272 & -3.162012 \\
$\mathrm{Y}$ & 0.551714 & -0.672359 & -0.540111 & -0.662383 \\
$\mathrm{Z}$ & 0.456266 & -0.373758 & -0.442735 & 0.364013 \\
\hline \multirow{2}{*}{ Magnitude } & 2.944198 & 3.236255 & 2.959844 & 3.251089 \\
& $(7.482864)$ & $(8.225146)$ & $(7.522630)$ & $(8.262847)$ \\
\hline
\end{tabular}

In au units, Debye units in parenthesis.

If the molecule is considered to be in a uniform electric field aligned along one of the axis of the system, the values of the polarizabilities along that axis $\left(\mu_{\mathrm{x}}, \alpha_{\mathrm{xx}}, \beta_{\mathrm{xxx}}, \gamma_{\mathrm{xxx}}\right)$ can be obtained by using eq.4. Tables $2 \mathrm{~A}$ and $2 \mathrm{~B}$ list the components of polarizability $\alpha$, where E4 and dipole are the contributors of polarizability to energy and dipole moment, respectively. The results show that the average polarizability increases as the polarization value increases. Note that the diagonal elements exhibit parallelism in sign irrespective of the method applied but it does not hold for the off-diagonal elements. 
Table 2A. Components of polarizability $\alpha$ (Applied electric field magnitude : 0.001). Components of polarizability

Method

\begin{tabular}{ccccc}
\hline & \multicolumn{2}{c}{ MNDO// B3LYP/CC-PVTZ } & \multicolumn{2}{c}{ PM3// B3LYP/CC-PVTZ } \\
\hline Field direction & E4 & Dipole & E4 & Dipole \\
\hline XX & 101.271402 & 77.834195 & 81.476646 & 81.469063 \\
YY & 91.634167 & 68.197510 & 68.203963 & 68.199057 \\
ZZ & 33.710802 & 10.277835 & 13.308350 & 13.306438 \\
XY & -2.575101 & -2.574591 & -4.646400 & -4.646921 \\
XZ & -0.295551 & -0.295574 & 0.699106 & 0.699903 \\
YZ & -0.639497 & -0.639470 & -0.946444 & -0.947303 \\
\hline $\begin{array}{l}\text { Average } \\
\text { polarizability }\end{array}$ & 75.538790 & 52.103180 & 54.329653 & 54.324853 \\
\hline
\end{tabular}

In au units.

Table 2B. Components of polarizability $\alpha$ (Applied electric field magnitude : 0.01). Components of polarizability

Method

\begin{tabular}{ccccc}
\hline & \multicolumn{2}{c}{ MNDO// B3LYP/CC-PVTZ } & \multicolumn{2}{c}{ PM3// B3LYP/CC-PVTZ } \\
\hline Field direction & E4 & Dipole & E4 & Dipole \\
\hline XX & 101.7738 & 78.31662 & 82.02514 & 82.00063 \\
YY & 92.02273 & 68.58571 & 68.59046 & 68.58603 \\
ZZ & 33.72485 & 10.2919 & 13.32381 & 13.32292 \\
XY & 2.624248 & 2.616245 & -4.70991 & -4.70272 \\
XZ & -0.30399 & -0.30407 & 0.703765 & 0.703272 \\
YZ & 0.647227 & 0.647182 & -0.98461 & -0.98452 \\
\hline Average & 75.840467 & 52.398077 & 54.646472 & 54.636526 \\
polarizability & & & & \\
\hline
\end{tabular}

In au units.

Note that in Tables 2-5 the calculations were performed on B3LYP/cc-PVTZ based optimized structure employing MNDO and PM3 single point calculations. 
The tensor components of the second order polarizability $(\beta)$ are presented in Tables $3 \mathrm{~A}$ and $3 \mathrm{~B}$.

Table 3A. Components of second order polarizability $(\beta)$ (Applied electric field magnitude : 0.001).

$$
\text { Method }
$$

\begin{tabular}{ccccc}
\hline & \multicolumn{2}{c}{ MNDO// B3LYP/CC-PVTZ } & \multicolumn{2}{c}{ PM3// B3LYP/CC-PVTZ } \\
\hline Components & E4 & Dipole & E4 & Dipole \\
\hline XXX & -91.410 & -91.692 & -213.595 & -213.338 \\
XYY & -2.402 & -2.545 & -39.188 & -38.300 \\
XZZ & 19.676 & 19.837 & 25.744 & 25.087 \\
YYY & 81 & 80.908 & 137.637 & 137.165 \\
YXX & 69.617 & 69.413 & 136.644 & 136.297 \\
YZZ & -0.629 & -0.553 & 1.545 & 2.027 \\
ZZZ & -1.989 & -1.946 & -6.933 & -7.587 \\
ZXX & -36.285 & -36.327 & -67.072 & -67.029 \\
ZYY & -5.295 & -5.336 & -11.387 & -11.317 \\
\hline
\end{tabular}

In au units.

Table 3B. Components of second order polarizability ( $\beta$ ) (Applied electric field magnitude : 0.01).

Method

MNDO// B3LYP/CC-PVTZ

PM3// B3LYP/CC-PVTZ

\begin{tabular}{ccccc}
\hline Components & E4 & Dipole & E4 & Dipole \\
\hline XXX & 110.502 & 123.21 & -227.681 & -236.962 \\
XYY & 4.102 & 2.377 & -41.699 & -40.298 \\
XZZ & -20.17 & -20.153 & 26.704 & 26.764 \\
YYY & 81.257 & 80.684 & 137.69 & 136.409 \\
YXX & 67.865 & 53.423 & 135.603 & 115.885 \\
YZZ & -0.838 & -0.89 & 1.012 & 0.867 \\
ZZZ & 2.06 & 2.064 & -6.979 & -6.995 \\
ZXX & 35.96 & 36.152 & -66.279 & -65.82 \\
ZYY & 4.666 & 4.67 & -10.848 & -10.861 \\
\hline
\end{tabular}

In au units. 
The vector components of $\beta$ and its value in the dipole moment are presented in Tables $4 \mathrm{~A}$ and $4 \mathrm{~B}$. As seen in Tables $4 \mathrm{~A}$ and $4 \mathrm{~B}$, the value of $\beta$ in dipole moment increases as the polarizability value increased ten times. Also note that in each method applied, the value of $\beta$ increases as the polarization increases. In the table components BI are expressed by the formula [2],

$$
\mathrm{BI}=(3 / 5)(\mathrm{BI} 11+\mathrm{BI} 22+\mathrm{BI} 33)
$$

Table 4A. Vector component BI of second order polarizability ( $\beta$ ). (Applied electric field magnitude : 0.001).

\begin{tabular}{ccccc}
\hline & \multicolumn{2}{c}{ MNDO// B3LYP/CC-PVTZ } & \multicolumn{2}{c}{ PM3// B3LYP/CC-PVTZ } \\
\hline BX & -44.482 & -44.641 & -136.224 & -135.931 \\
BY & 89.993 & 89.861 & 165.496 & 165.294 \\
BZ & -26.141 & -26.165 & -51.235 & -51.56 \\
\hline $\begin{array}{l}\text { Value of } \beta \\
\text { dipole moment. }\end{array}$ & 22.239 & 22.414 & 92.024 & 91.744 \\
\hline
\end{tabular}

In au, esu units in parenthesis.

Table 4B. Vector component BI of second order polarizability ( $\beta$ ). (Applied electric field magnitude : 0.01).

\begin{tabular}{ccccc}
\hline & \multicolumn{2}{c}{ MNDO// B3LYP/CC-PVTZ } & \multicolumn{2}{c}{ PM3// B3LYP/CC-PVTZ } \\
\hline BX & 56.661 & 63.26 & -145.606 & -150.298 \\
BY & 88.97 & 79.93 & 164.583 & 151.897 \\
BZ & 25.612 & 25.732 & -50.464 & -50.206 \\
\hline Value of $\beta$ in & 34.995 & 43.040 & 102.434 & 109.611 \\
dipole moment. & $\left(0.30310^{-30}\right)$ & $\left(0.37310^{-30}\right)$ & $\left(0.88710^{-30}\right)$ & $\left(0.94910^{-30}\right)$ \\
\hline
\end{tabular}

In au, esu units in parenthesis.

Tables 5A and 5B show the third order polarizability $(\gamma)$ vector components involved in the calculation of averaged $\gamma$ values as the applied polarizability value increased. The average value of $\gamma$ is expressed as [2],

Average Gamma $=(1 / 5)[X X X X+Y Y Y Y+Z Z Z Z+2(X X Y Y+X X Z Z+Y Y Z Z)](10)$

As seen in the tables $\gamma$ strongly responds to the increase of polarizability. 
Table 5A. Third order $(\gamma)$ polarizability (Applied electric field magnitude : 0.001).

\begin{tabular}{ccccc} 
& \multicolumn{2}{c}{ MNDO// B3LYP/CC-PVTZ } & \multicolumn{2}{c}{ PM3// B3LYP/CC-PVTZ } \\
\hline $\begin{array}{c}\text { Vector } \\
\text { component }\end{array}$ & E4 & Dipole & E4 & Dipole \\
\hline XXXX & 19036.53 & 18511.04 & 19645.04 & 21602 \\
YYYY & -1182.64 & -1416.87 & 807.502 & 277.544 \\
ZZZZ & 28.791 & 103.402 & 144.857 & 603.88 \\
XXYY & 4074.776 & 3849.748 & 6465.778 & 5073.069 \\
XXZZ & 478.655 & 485.795 & 980.709 & 1609.056 \\
YYZZ & 193.231 & 137.359 & 465.796 & 1217.813 \\
\hline Value of $\gamma$ & 5475.20 & 5228.67 & 7284.39 & 7656.66 \\
& $\left(2.7656110^{-36}\right)$ & $\left(2.6410910^{-36}\right)$ & $\left(3.6794610^{-36}\right)$ & $\left(3.8675010^{-36}\right)$ \\
\hline
\end{tabular}

In au units. see units in parenthesis.

Table 5B. Third order $(\gamma)$ polarizability (Applied electric field magnitude : 0.01). MNDO// B3LYP/CC-PVTZ PM3// B3LYP/CC-PVTZ

\begin{tabular}{ccccc}
\hline $\begin{array}{c}\text { Vector } \\
\text { component }\end{array}$ & E4 & Dipole & E4 & Dipole \\
\hline XXXX & 20689.91 & 21443.4 & 21134.63 & 21866.37 \\
YYYY & -1188.39 & -1181.03 & 764.001 & 759.483 \\
ZZZZ & 40.3 & 40.382 & 147.984 & 148.085 \\
XXYY & 4191.187 & 4184.982 & 6592.939 & 6583.735 \\
XXZZ & 480.476 & 480.259 & 959.381 & 958.45 \\
YYZZ & 202.522 & 202.355 & 472.516 & 471.987 \\
\hline Value of $\gamma$ & 5858.04 & 6007.59 & 7619.26 & 7760.46 \\
& $\left(2.9589910^{-36}\right)$ & $\left(3.0345310^{-36}\right)$ & $\left(3.8486110^{-36}\right)$ & $\left(3.9199310^{-36}\right)$ \\
\hline
\end{tabular}

In au units. esu units in parenthesis.

Figure 3 shows the axes of inertia of ANTA molecule. In the figure, $\mathrm{X}$-axis is along the longest principle axis of the molecule whereas $\mathrm{Y}$ axis is perpendicular to $\mathrm{X}$-axis. The $\mathrm{Z}$-axis is perpendicular to XY-plane. 


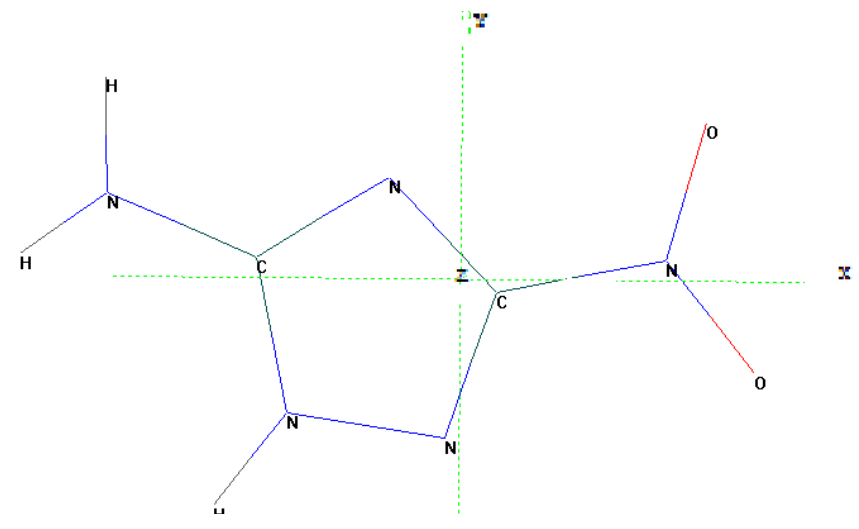

Figure 3. ANTA molecule and its axes of inertia.

Table 6 shows the effect of field applied along the axes of inertia (X,Y and Z) of ANTA molecule and strength on the dipole and its components. The components of the dipole are designated as $\mathrm{x}, \mathrm{y}$ and $\mathrm{z}$. The calculations have been performed at the level of MNDO//B3LYP/cc-PVTZ.

Table 6. Effect of field direction and strength on dipole and its components of ANTA molecule.

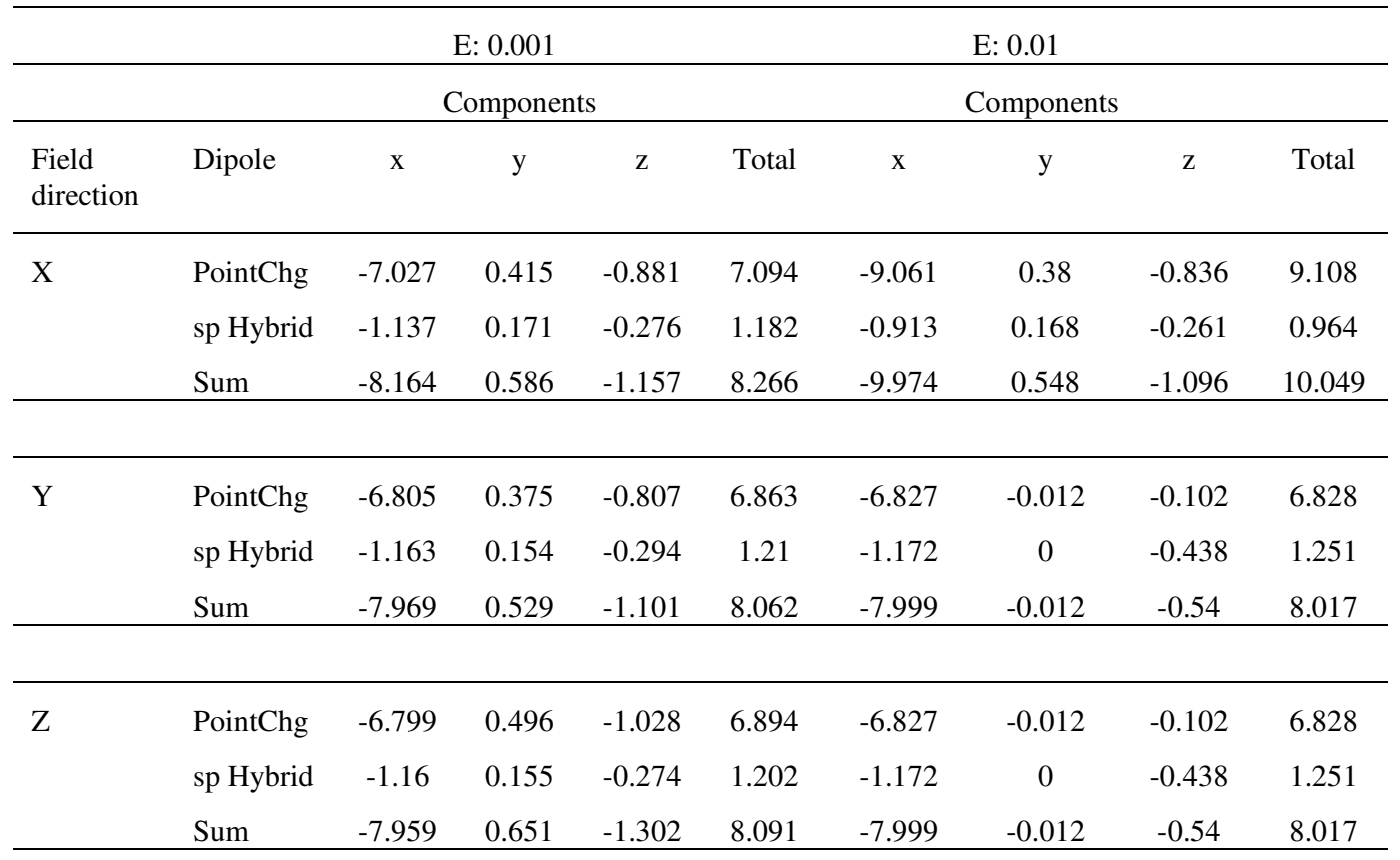

Dipole in Debyes. E: Field strength in au units. 
As seen in Table 6, the total value of the dipole moment increases if the field strength (F) is increased in the direction of the $\mathrm{X}$-axis along which the donor $\left(\mathrm{NH}_{2}\right)$ and acceptor $\left(\mathrm{NO}_{2}\right)$ groups reside. The applied electric field perturbs the electron population in such a way that the value of dipole moment vector increases as the field strength is increased. However, increase of the field strength along the $\mathrm{Y}$ or $\mathrm{Z}$ axes slightly decreases the dipole moment value.

Figure 4 displays the effect of electric field on the molecular orbital energy spectra of

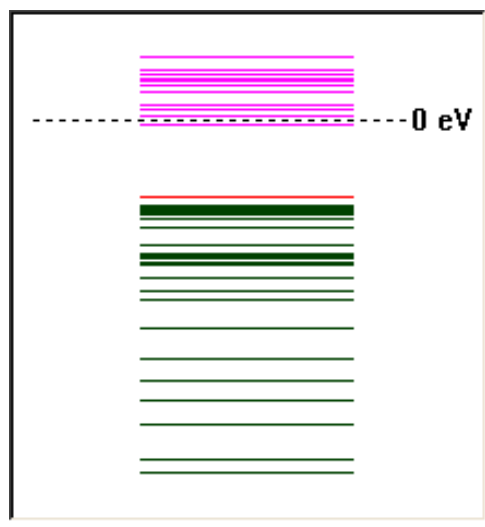

$$
\mathrm{E}_{\mathrm{X}}=0.001
$$
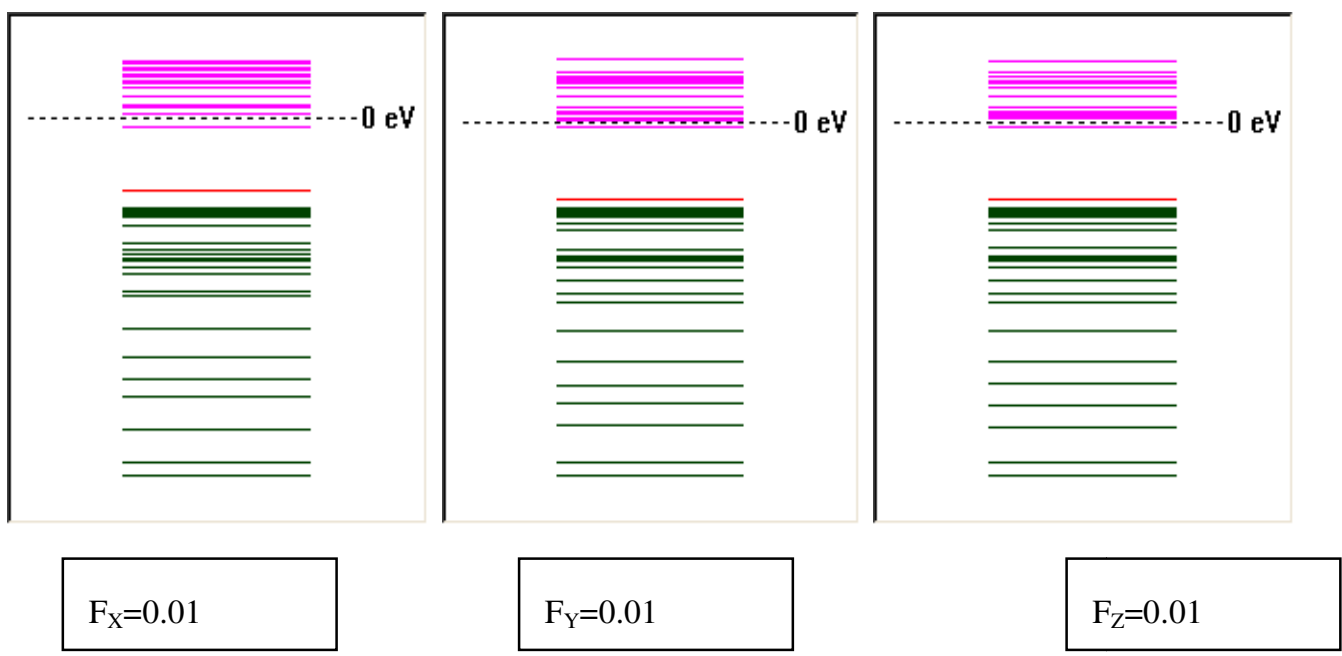

Figure 4. Effect of electric field on the molecular orbital energy spectra of ANTA molecule (Applied field in au units, MNDO// B3LYP/cc-PVTZ)). 
ANTA molecule. In the case of F: 0.001 the distribution of molecular orbital energy levels does not exhibit discernable perturbation depending on the field direction. Whereas when $F$ value is set to $0.01 \mathrm{au}$, the distribution standing for $\mathrm{F}_{\mathrm{x}}$ case is different from the others.

Table 7 shows the effect of field strength on the HOMO, LUMO energies and the interfrontier molecular orbital energy gap $(\Delta \varepsilon)$ value of ANTA molecule. Note that $\Delta \varepsilon=$ $\varepsilon_{\text {LUMO }}-\varepsilon_{\text {HOMO }}$. As seen in the table, as the field in $\mathrm{X}, \mathrm{Y}$ and Z-directions increases the HOMO energy level raises up. Again the greatest effect occurs in case of $X$-direction. On the contrary, the LUMO energy decreases as the field strength increases in X, Y and Zdirections in all the cases. Consequently, the interfrontier molecular orbital energy gap value decreases as the field strength increases, keeping the direction be the same. The electron distribution can be distorted readily if the LUMO energy level lies close to the HOMO energy, so the polarizability is then large. If the LUMO lies high above the HOMO, an applied field cannot perturb the electron distribution significantly and the polarizability is low [34]. Note that the impact sensitivity of an explosive is related to $\Delta \varepsilon$ value such that decrease of it increases the sensitivity [35, 36]. Also note that in the case of ANTA the decrease in $\Delta \varepsilon$ is greater if the field direction lies along the $\mathrm{X}$-axis.

Table 7. Effect of field strength on the HOMO, LUMO energies and $\Delta \varepsilon$ value of ANTA molecule.

\begin{tabular}{lccc}
\hline Field direction & HOMO & LUMO & $\Delta \varepsilon$ \\
\hline $\mathrm{X}$ & -10.315015 & -0.930985 & 9.38403 \\
& -9.722733 & -1.29873 & 8.424003 \\
\hline $\mathrm{Y}$ & -10.375451 & -0.901036 & 9.474415 \\
& -10.358150 & -0.920074 & 9.438076 \\
\hline $\mathrm{Z}$ & -10.378779 & -0.894343 & 9.484436 \\
& -10.358150 & -0.920074 & 9.438076 \\
\hline
\end{tabular}

The first and second entries in each row have E values of 0.001 and 0.01 in $\mathrm{eV}$, respectively. 
Figures 5 and 6 show the HOMO and LUMO patterns of ANTA molecule in two different field strengths. As seen in the figures the main effect of the increased field occurs on the LUMO pattern (on the carbon atom having the amino group) when it is set along the $\mathrm{X}$ - and $\mathrm{Z}$-axes.
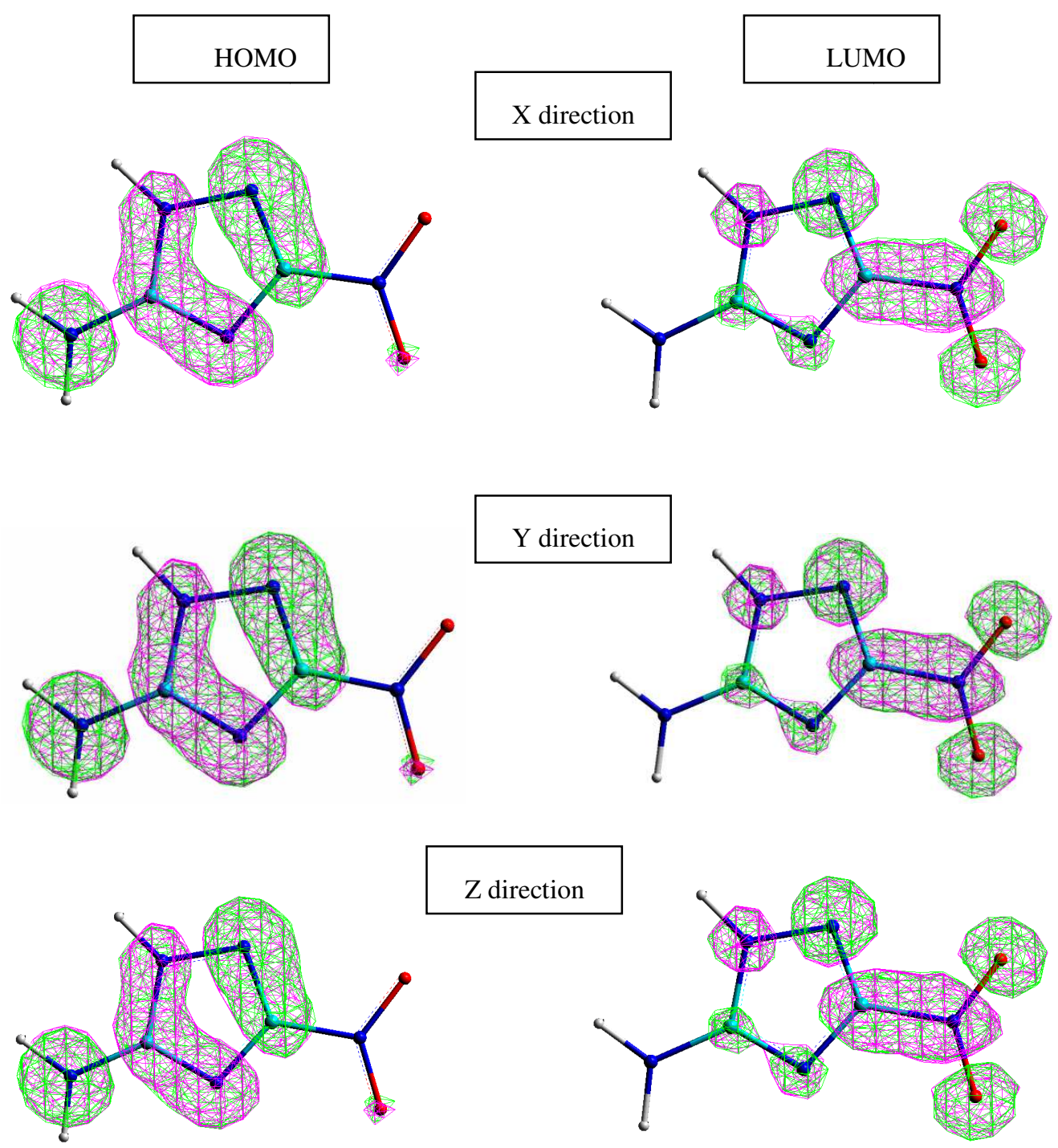

Figure 5. The HOMO and LUMO patterns of ANTA in F: 0.001 au. (MNDO//B3LYP/cc-PVTZ). 

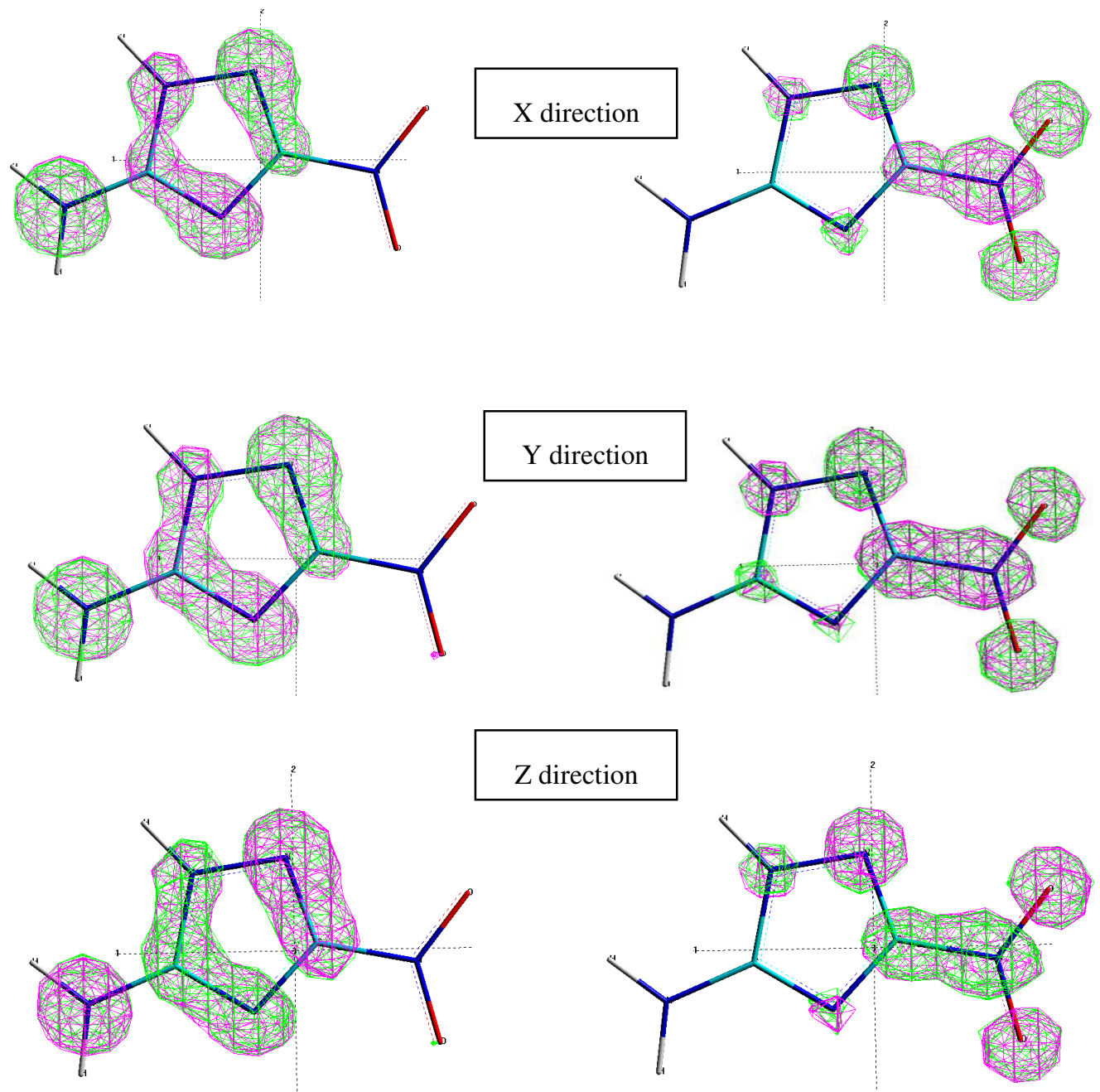

Figure 6. The HOMO and LUMO patterns of ANTA in F: 0.01 au. (MNDO//B3LYP/ccPVTZ).

\section{Conclusion}

The present study on ANTA molecule has revealed that the applied electric field mainly affects ANTA when the direction of the field is set along the principle axis of the molecule. Then the HOMO energy level raises up whereas the LUMO level decreases. Meanwhile the interfrontier molecular orbital energy gap decreases as the magnitude of the field increases. So, the impact sensitivity of ANTA may increase by exposing it to an electric field. Obviously, $a b$ initio methods are expected to yield reliable and 
quantitatively correct results. Note that semi empirical calculations tend to be qualitative unless the structure optimization is done by means of $a b$ initio/DFT methods at high level basis sets.

\section{References}

[1] D.C. Young, Computational Chemistry, New York: Wiley-Interscience, 2001.

[2] H.A. Kurtz, J.J.P. Stewart and K.M. Dieter, Calculation of the nonlinear optical properties of molecules, J. Computational Chemistry 11(1) (1990), 82-87. https://doi.org/10.1002/jcc.540110110

[3] P.W. Atkins, Quanta, Oxford Chemistry Series, Oxford: Clarendon Press, 1974.

[4] A. Hinchliffe and R.W. Munn, Molecular Electromagnetism, Chichester: Wiley, 1985.

[5] V. Kondratyev, The Structure of Atoms and Molecules, Moscow: Mir, 1967.

[6] V. Librando, A. Alparone and Z. Minniti, Computational study on dipole moment, polarizability and second hyperpolarizability of nitronaphthalenes, J. Molecular Structure (Theochem) 856 (2008), 105-111. https://doi.org/10.1016/j.theochem.2008.01.022

[7] D.P. Shelton, Dispersion of the nonlinear susceptibility measured for benzene, J. Opt. Soc. Am. B 2 (1985), 1880-1882. https://doi.org/10.1364/JOSAB.2.001880

[8] S. Millefiori and A. Alparone, Ab initio study of the molecular structure, polarizability and first hyperpolarizability of 6-hydroxy-1-formylfulvene, J. Chem. Soc., Faraday Trans. 90 (1994), 2873-2879. https://doi.org/10.1039/FT9949002873

[9] R. Zaleśny, I.W. Bulik, W. Bartkowiak, J.M. Luis, A. Avramopoulos, M.G. Papadopoulos and P. Krawczyk, Electronic and vibrational contributions to first hyperpolarizability of donor-acceptor-substituted azobenzene, J. Chem. Phys. 133 (2010), 244-308. https://doi.org/10.1063/1.3516209

[10] I. Harczuk, O. Vahtras and H. Ågren, Hyperpolarizabilities of extended molecular mechanical systems, Phys. Chem. Chem. Phys. 18 (2016), 8710-8722.

https://doi.org/10.1039/c5cp06688c

[11] W. Bartkowiak, R. Zaleśny, W. Niewodniczański and J. Leszczynski, Quantum chemical calculations of the first- and second-order hyperpolarizabilities of molecules in solutions, J. Phys. Chem. A 105(47) (2001), 10702-10710. https://doi.org/10.1021/jp010682s

[12] D. Paschoal and H.F. Dos Santos, Assessing the quantum mechanical level of theory for prediction of linear and nonlinear optical properties of push-pull organic molecules, Mol. Model 19 (2013), 2079-2090. https://doi.org/10.1007/s00894-012-1644-4 
[13] J.P. Agrawal, High Energy Materials, Weinheim: Wiley-VCH, 2010. https://doi.org/10.1002/9783527628803

[14] K.Y. Lee, C.B. Storm, M.A. Hiskey and M.D. Coburn, An improved synthesis of 5amino-3-nitro-1H-1,2,4-triazole (ANTA), a useful intermediate for the preparation of insensitive high explosives, J. Energ. Mat. 9(5) (1991), 415-428.

https://doi.org/10.1080/07370659108019382

[15] R.L. Simpson, P.F. Pagoria, A.R. Mitchell and C.L. Coon, Synthesis, properties and performance of the high explosive ANTA, Prop. Explos. Pyrotech. 19 (1994), 174-179. https://doi.org/10.1002/prep.19940190405

[16] T.D. Manship, D.M. Smith and D.G. Piercey, An improved synthesis of the insensitive energetic material 3-amino-5-nitro-1,2,4-triazole (ANTA), Prop. Explos. Pyrotech. 45(10) (2020), 1621-1626. https://doi.org/10.1002/prep.202000097

[17] J.F. Moxnes, $\varnothing$. Frøyland and T. Risdal, A computational study of ANTA and NTO derivatives, Journal of Molecular Modeling 23(8) (2017), 240.

https://doi.org/10.1007/s00894-017-3408-7

[18] J.J.P. Stewart, Optimization of parameters for semi empirical methods I, J. Comput Chem. 10 (1989), 209-220. https://doi.org/10.1002/jcc.540100208

[19] J.J.P. Stewart, Optimization of parameters for semi empirical methods II, J. Comput. Chem. 10 (1989), 221-264. https://doi.org/10.1002/jcc.540100209

[20] A.R. Leach, Molecular Modeling, Essex: Longman, 1997.

[21] W. Kohn and L.J. Sham, Self-consistent equations including exchange and correlation effects, Phys. Rev. 140 (1965), 1133-1138. https://doi.org/10.1103/PhysRev.140.A1133

[22] R.G. Parr and W. Yang, Density Functional Theory of Atoms and Molecules, London: Oxford University Press, 1989.

[23] A.D. Becke, Density-functional exchange-energy approximation with correct asymptotic behavior, Phys. Rev. A 38 (1988), 3098-3100. https://doi.org/10.1103/PhysRevA.38.3098

[24] S.H. Vosko, L. Vilk and M. Nusair, Accurate spin-dependent electron liquid correlation energies for local spin density calculations: a critical analysis, Can. J. Phys. 58 (1980), 1200-1211. https://doi.org/10.1139/p80-159

[25] C. Lee, W. Yang and R.G. Parr, Development of the Colle-Salvetti correlation-energy formula into a functional of the electron density, Phys. Rev. B 37 (1988), 785-789. https://doi.org/10.1103/PhysRevB.37.785

[26] SPARTAN 06, Wavefunction Inc., Irvine CA, USA, 2006. 
[27] Hyperchem 7.0, Hypercube Inc., Waterloo, Ontario, Canada, 2002.

[28] L.V. Interrante and M.S. Hampton-Smith, Chemistry of Advanced Materials, New York: Wiley-VCH, 1998.

[29] D. Lu, G. Chen, J.W. Perry and W.A. Goddard, Valence-bond charge-transfer model for nonlinear optical properties of charge-transfer organic molecules, J. Am. Chem. Soc. 116(23) (1994), 10679-10685. https://doi.org/10.1021/ja00102a037

[30] R. Marder, D.N. Beratan, L.T. Cheng, Approaches for optimizing the first electronic hyperpolarizability of conjugated organic molecules, Science 252 (5002) (1991), 103 106. https://doi.org/10.1126/science.252.5002.103

[31] S.R. Marder, C.B. Gorman, F. Meyers, J.W. Perry, G. Bourhill, J.-Luc. Brédas and B.M. Pierce, A unified description of linear and nonlinear polarization in organic polymethine dyes, Science 265(5172) (1994), 632-635. https://doi.org/10.1126/science.265.5172.632

[32] F. Meyers, S.R. Marder, B.M. Pierce and J.L. Bredas, Investigation of the relationship between molecular polarizabilities (alpha, beta and gamma) and bond length alternation, J. Am. Chem. Soc. 116(23) (1994), 10703-10714. https://doi.org/10.1021/ja00102a040

[33] C.B. Gorman and S.R. Marder, An investigation of the interrelationships between linear and nonlinear polarizabilities and bond-length alternation in conjugated organic molecules, Proc. Natl. Acad. Sci., USA 90 (1993), 11297-11301.

https://doi.org/10.1073/pnas.90.23.11297

[34] P. Atkins and J. de Paula, Atkins' Physical Chemistry, $7^{\text {th }}$ ed., Oxford: Oxford University Press, 2002.

[35] V. Anbu, K.A. Vijayalakshmi, R. Karunathan, A.D. Stephen and P.V. Nidhin, Explosives properties of high energetic trinitrophenyl nitramide molecules: A DFT and AIM analysis, Arab. J. Chem. 12(5) (2019), 621-632.

https://doi.org/10.1016/j.arabjc.2016.09.023

[36] N.R. Badders, C. Wei, A.A. Aldeeb, W.J. Rogers and M.S. Mannan, Predicting the impact sensitivities of polynitro compounds using quantum chemical descriptors, $J$. Energ. Mater. 24 (2006), 17-33. https://doi.org/10.1080/07370650500374326 